University of Texas at El Paso

ScholarWorks@UTEP

$10-2004$

\title{
Convergence Properties of an Interval Probabilistic Approach to System Reliability Estimation
}

\author{
Cliff Joslyn \\ Vladik Kreinovich \\ The University of Texas at El Paso, vladik@utep.edu
}

Follow this and additional works at: https://scholarworks.utep.edu/cs_techrep

Part of the Computer Engineering Commons

Comments:

UTEP-CS-02-29b.

Preliminary version published as Los Alamos National Laboratory Technical Report LA-

UR-02-6261, Los Alamos, NM, 2002; full paper published in International Journal of General

Systems, 2005, Vol. 34, No. 4, pp. 465-482.

\section{Recommended Citation}

Joslyn, Cliff and Kreinovich, Vladik, "Convergence Properties of an Interval Probabilistic Approach to System Reliability Estimation" (2004). Departmental Technical Reports (CS). 359.

https://scholarworks.utep.edu/cs_techrep/359

This Article is brought to you for free and open access by the Computer Science at ScholarWorks@UTEP. It has been accepted for inclusion in Departmental Technical Reports (CS) by an authorized administrator of ScholarWorks@UTEP.For more information, please contact Iweber@utep.edu. 


\title{
Convergence Properties of an Interval Probabilistic Approach to System Reliability Estimation
}

\author{
Cliff Joslyn* and Vladik Kreinovich ${ }^{\dagger}$
}

October, 2004

\begin{abstract}
Based on a black box model of a complex system, and on intervals and probabilities describing the known information about the inputs, we want to estimate the system's reliability. This problem is motivated by a number of problem areas, most specifically in engineering reliability analysis under conditions of poor measurement and high complexity of system models. Using the results of tests performed on the system's computer model, we can estimate the lower and upper bounds of the probability that the system is in a desirable state. This is equivalent to using Monte-Carlo sampling to estimate cumulative belief and plausibility values of functionally propagated finite random intervals. In this paper, we prove that these estimates are correct in the sense that under reasonable assumptions, these estimates converge to the actual probability bounds.
\end{abstract}

Keywords: Interval probability, interval analysis, reliability analysis, Dempster-Shafer evidence theory, random sets, random intervals, epistemic uncertainty, Monte-Carlo sampling.

\section{Introduction}

In this paper, we consider the problem of modeling the risk and reliability of complex technical system. The behavior of this system is determined by the values of the corresponding parameters $x=\left\langle x^{(1)}, x^{(2)}, \ldots, x^{(n)}\right\rangle$; for example, for a nuclear reactor, these characteristics could include the

${ }^{*}$ Knowledge Systems and Computational Biology Team, Modeling, Algorithms, and Informatics Group (CCS-

3), Mail Stop B265, Los Alamos National Laboratory, Los Alamos, NM 87545, USA, joslyn@lanl.gov, http://www.c3.lanl.gov/ joslyn, (505) 667-9096.

${ }^{\dagger}$ Department of Computer Science, University of Texas at El Paso, 500 W. University, El Paso, TX 79968, USA, vladik@cs.utep.edu 
thicknesses of the walls, the locations of the radiation absorbers, etc. For each combination $x$ of these parameters, the system exhibits certain characteristics $y=\left\langle y^{(1)}, y^{(2)}, \ldots, y^{(m)}\right\rangle$; e.g., for a nuclear reactor, the list of such characteristics include neutron flux, temperature, etc.

We assume that the parameters set is complete (or almost complete), so that the observed state is uniquely determined by the values $x$ of the parameters, so that $y=f(x)$ for some function $f$. In this context, $f$ acts as a model of our knowledge of the system.

The reliability of such a system is related to the fact that some states $y$ are desirable, while some other states are not. Thus we'll be concerned with the conditions under which the system output occurs within some desirable output set $B_{0}$.

If we knew the exact values of the parameters $x^{(i)}$, then we would be able to determine the corresponding state $y=f(x)$ and check whether it is desirable, $f(x) \in B_{0}$, or not. But in real life, we usually do not know the exact values of $x^{(i)}$. Instead, in some instances we may know a lower bound $\underline{x}^{(i)}$ and the upper bound $\bar{x}^{(i)}$ that are known to contain $x^{(i)}$. Often, we do not know how more or how less probable are different values within this interval, i.e., what is an actual probability distribution for $x^{(i)}$ within this interval.

In some cases, instead of a single interval, several experts provide different intervals corresponding to different possible situations. Then, in addition to these intervals, we usually also know the probability of each such situation. For example, a reactor shell can come from three different manufacturing plants, and we know the frequencies with which they come from different plants, i.e., the probabilities that a randomly selected shell is from this particular plant. For each plant, we also know the interval of possible value of thickness for shells produced by this plant.

Furthermore, systems of interest are characterized by a high complexity such that these models $f$ are large simulation codes. These codes are sometimes so huge that each run requires days on supercomputers. As a result, we cannot typically control what inputs we feed into the code, but have to reply on the results of the testing, i.e., on some pairs $\left\{\left\langle x_{1}, y_{1}\right\rangle,\left\langle x_{2}, y_{2}\right\rangle, \ldots,\left\langle x_{M}, y_{M}\right\rangle\right\}$ corresponding to these actual test runs.

Given such information, what can you know about the probability $P$ that the resulting state is desirable? If we knew the joint probability distribution of the parameters, then we could determine the probability of different values of $y$ and thus we could get the probability $\operatorname{Pr}\left(f(x) \in B_{0}\right)$. In reality, we only have partial information about the probability distributions. For different distrib- 
utions, we may get different values of $P$. Our goal is, therefore, to find the interval $\mathbf{P}=[\underline{P}, \bar{P}]$ of possible values of this probability $P$.

In this paper we develop the methodology of this problem formulation under conditions of only sampling information from $f(x)$, and determine convergence conditions for both the upper and lower value of this probability interval.

This class of problems is quite realistic in certain engineering modeling contexts, and was the recent focus of a major interdisciplinary research effort among the engineering modeling, risk analysis, and generalized information theoretical and imprecise probability communities [2, 7, 11]. Most specifically, this can be understood as the problem of propagating a finite random interval [8] through the model $f$. Monte-Carlo sampling approaches to such random interval sampling are in development $[5,6,9]$, and this work stands to assist that effort in providing a rigorous formulation of some of the required convergence results.

\section{Probability Intervals on Input Information}

We begin by introducing our basic mathematical constructs, characterizing the uncertainty structures on the inputs, and demonstrating the resulting probability intervals.

\section{$2.1 \quad$ Input Information}

Let $\mathbb{R}$ be the real numbers, and $\mathbb{N}:=\{1,2, \ldots\}, \mathbb{N}_{n}:=\{1,2, \ldots, n\}$. Assume integers $n, m \in \mathbb{N}$ where $n$ is the number of inputs and $m$ the number of outputs, and let $f: \mathbb{R}^{n} \mapsto \mathbb{R}^{m}$.

Let the $i$ 'th input be denoted $x^{(i)}$, taking values on a set $X^{(i)}=\mathbb{R}$, for $i \in \mathbb{N}_{n}$. Similarly, let the $l$ 'th output be denoted $y^{(l)}$, taking values on a set $Y^{(l)}=\mathbb{R}$, for $l \in \mathbb{N}_{m}$. Let $x:=\left\langle x^{(1)}, x^{(2)}, \ldots, x^{(n)}\right\rangle \in$ $\mathbb{R}^{n}$ be called input points, and $y:=\left\langle y^{(1)}, y^{(2)}, \ldots, y^{(m)}\right\rangle \in \mathbb{R}^{m}$ be called states.

Let $X$ and $Y$ be the sets of closed, rectangular boxes in $\mathbb{R}^{n}$ and $\mathbb{R}^{m}$ respectively, and generically, let $A \in X, B \in Y$. More specifically, let $B_{0} \in Y$ be the box of desired states; all other states $y \in \mathbb{R}^{m}, y \notin B_{0}$ will be called undesirable.

Let $A^{(i)}=\left[\underline{x}^{(i)}, \bar{x}^{(i)}\right] \subseteq X^{(i)}$ be a closed interval of the $i$ th input $X^{(i)}$, and $B^{(l)}=\left[\underline{y}^{(l)}, \bar{y}^{(l)}\right] \subseteq Y^{(l)}$ a closed interval of the $l^{\prime}$ th output $Y^{(l)}$. We assume that for every input $i$ from 1 to $n$, we have 
information about $i$-th input expressed as a finite collection of $N^{(i)} \in \mathbb{N}$ weighted intervals. In particular, denote the uncertainty structure on the $i$ 'th input as

$$
\mathcal{S}^{(i)}:=\left\{\left\langle A_{1}^{(i)}, p_{1}^{(i)}\right\rangle, \ldots,\left\langle A_{j^{(i)}}^{(i)}, p_{j^{(i)}}^{(i)}\right\rangle, \ldots,\left\langle A_{N^{(i)}}^{(i)}, p_{N^{(i)}}^{(i)}\right\rangle\right\}
$$

where $j^{(i)} \in \mathbb{N}_{N^{(i)}}, A_{j^{(i)}}^{(i)}=\left[\bar{x}_{j^{(i)}}^{(i)}, \underline{x}_{j^{(i)}}^{(i)}\right] \subseteq X^{(i)}$ is one such interval, $p_{j^{(i)}}^{(i)} \in[0,1]$, and we have $\forall i \in \mathbb{N}_{n}$ the probabilistic normalization criterion

$$
\sum_{j^{(i)}=1}^{N^{(i)}} p_{j^{(i)}}^{(i)}=1 .
$$

So in general, across the different inputs $X^{(i)}$, we can choose a particular combination of intervals $A_{j^{(i)}}^{(i)}$, one for each input dimension $X^{(i)}$. Denote $\vec{j}:=\left\langle j^{(1)}, j^{(2)}, \ldots, j^{(n)}\right\rangle$ as indicating these combinations. There are $N:=\prod_{i=1}^{n} N^{(i)}$ such possible combinations. Since there exists a bijective mapping between $\mathbb{N}_{N}$ and the set of all combinations $\vec{j}$, we can thereby use the $j \in \mathbb{N}_{N}$ to enumerate the various possible $\vec{j}$. Also, denote $j^{(i)} \in \vec{j}$ to indicate that a particular $j^{(i)}$ is one of the components of $\vec{j}$.

So for each such combination $\vec{j}$, we can define the box $A_{\vec{j}}$ as the Cartesian product of the corresponding intervals:

$$
A_{\vec{j}}:=\underset{i=1}{\stackrel{n}{X}} A_{j^{(i)}}^{(i)} \in X
$$

also denoted $A_{j} \in X$ as appropriate. Furthermore, assume that the information corresponding to different parameters are independent. Then for each combination $\vec{j}$, we have the overall probability "mass" $p_{\vec{j}}:=\prod_{i=1}^{n} p_{j(i)}^{(i)}$, also denoted $p_{j}$. In this way, from the individual uncertainty structures $\mathcal{S}^{(i)}$ we can construct the overall input uncertainty structure

$$
\mathcal{S}:=\left\{\left\langle A_{\vec{j}}, p_{\vec{j}}\right\rangle\right\}_{\vec{j}}=\left\{\left\langle A_{1}, p_{1}\right\rangle, \ldots,\left\langle A_{j}, p_{j}\right\rangle, \ldots,\left\langle A_{N}, p_{N}\right\rangle\right\}
$$

An example is shown in Fig. 1 for $n=2$ input parameters and $N^{(1)}=N^{(2)}=2$ intervals on each input parameter. The input intervals $A_{1}^{(1)}, A_{2}^{(1)}$ on $X^{(1)}$ and $A_{1}^{(2)}, A_{2}^{(2)}$ on $X^{(2)}$ are shown, with probabilities

$$
p_{1}^{(1)}=.4, \quad p_{2}^{(1)}=.6, \quad p_{1}^{(2)}=.2, \quad p_{2}^{(2)}=.8,
$$

which assignment satisfies (1). The boxes $A_{\vec{j}}$ and masses $p_{\vec{j}}$ are shown, along with their enumerated forms $A_{j}, p_{j}$. The bijective mapping $\mathbb{N}_{N} \leftrightarrow\{\vec{j}\}$ is

$$
\langle 1,\langle 1,1\rangle\rangle, \quad\langle 2,\langle 1,2\rangle\rangle, \quad\langle 3,\langle 2,1\rangle\rangle, \quad\langle 4,\langle 2,2\rangle\rangle
$$


shown as tuples of the form $\left\langle j, \vec{j}=\left\langle j^{(1)}, j^{(2)}\right\rangle\right\rangle$.

Note that for illustrative purposes, in Fig. 1 we show the boxes $A_{j^{(i)}}^{(i)}$ slightly offset from each other, in order to clearly distinguish them. In fact, their borders overlap where they are shown very close to each other.

\subsection{Consistent Probability Measures}

Consider a probability measure $P$ on $\mathbb{R}^{n}$. For an arbitrary box in the input space $A \in X$, we say that the total probability $P(A)$ is consistent with the input uncertainty structure $\mathcal{S}$ if there exists a collection of total probabilities $\left\langle P_{j}(A)\right\rangle$, which are concentrated on the corresponding boxes $A_{j}$, such that

$$
P(A)=\sum_{j=1}^{N} p_{j} P_{j}(A)
$$

An example is also shown in Fig. 1, for $A \in X$ as illustrated. It can be demonstrated that $P(A)=.2$ is consistent with $\mathcal{S}$, because for the distribution of total probabilities $\left\langle P_{j}(A)\right\rangle=\langle .5, .5,0,0\rangle$ we have

$$
\sum_{j=1}^{N} p_{j} P_{j}(A)=.08 \cdot .5+.32 \cdot .5+.48 \cdot 0+.12 \cdot 0=.2=P(A)
$$

\subsection{Probability Intervals}

Our goal is to describe the smallest interval $\mathbf{P}:=[\underline{P}, \bar{P}]$ that contains all possible values of $\operatorname{Pr}\left(f(x) \in B_{0}\right)=P\left(\left\{x: f(x) \in B_{0}\right\}\right)$ for all consistent probability measures $P(A)$.

\section{Theorem 1}

$$
\underline{P}=\sum_{j: f\left(A_{j}\right) \subseteq B_{0}} p_{j}, \quad \bar{P}=\sum_{j: f\left(A_{j}\right) \cap B_{0} \neq \emptyset} p_{j} .
$$

\subsection{Comments}

- Proofs of all theorems, corollaries, and propositions can be found in the Appendix.

- The problem formulation, including a different version of Theorem 1, was originally expressed by Joslyn and Helton [9]. 
- Many readers will recognize (2) as formulae from the Dempster-Shafer theory $[4,13]: \underline{P}$ is the formula for belief, and $\bar{P}$ is the formula for plausibility, with boxes $A_{j}$ as focal elements, and with $p_{j}$ being the mass of the "basic probability assignment" of the corresponding box $A_{j}$. The formula for the mass of the box is also familiar: it corresponds to the Dempster-Shafer combination of the corresponding "knowledge bases" $\mathcal{S}^{(i)}$. This similarity is no accident: Dempster-Shafer formalism was originally designed to describe exactly such situations - when we have only partial information about probabilities.

- In particular, we assume a random set interpretation of a Dempster-Shafer structure [1, 12], so that the weights $p_{j^{(i)}}^{(i)}$ are interpreted as values of a discrete probability density over the atomic events which are actually the intervals $A_{j(i)}^{(i)}$, and thus which may be overlapping, included within each other, or disjoint.

- Similarly, our formulation of the input uncertainty structure, both the dimensional form $\mathcal{S}^{(i)}$ and the overall form $\mathcal{S}$, is isomorphic to a formulation as a finite random set [3, 9], which is itself isomorphic to Dempster-Shafer evidence theory.

\section{Basic Sampling Results}

Theorem 1 describes how we can compute the bounds $\underline{P}$ and $\bar{P}$ in the ideal situation when we know the function $f(x)$. In reality, all we know are some samples $\left\langle x_{k}, f\left(x_{k}\right)\right\rangle$ from this function.

Denote $y_{k}=f\left(x_{k}\right)$, and let $S:=\left\{\left\langle x_{1}, y_{1}\right\rangle, \ldots,\left\langle x_{k}, y_{k}\right\rangle, \ldots\right\}$ be an infinite sequence of sample points for $k \in \mathbb{N}$. For some $M \in \mathbb{N}$, denote $S_{M}:=\left\{\left\langle x_{1}, y_{1}\right\rangle, \ldots,\left\langle x_{M}, y_{M}\right\rangle\right\}$ as the initial finite subsequence of $M$ sample points.

How can we estimate $\underline{P}$ and $\bar{P}$ based on these samples?

\subsection{Lower Probability}

Let us start with $\underline{P}$. According to Proposition 1, the actual value $\underline{P}$ is the sum of the values $p_{j}$ for all the boxes $A_{j}$ for which $f\left(A_{j}\right) \subseteq B_{0}$. This set theoretical condition can be re-expressed in logical terms:

$$
f\left(A_{j}\right) \subseteq B_{0} \equiv\left\{f(x): x \in A_{j}\right\} \subseteq B_{0}
$$


Interval Probabilistic Approach

$$
\begin{aligned}
& \equiv \forall x \in A_{j}, f(x) \in B_{0} \\
& \equiv x \in A_{j} \rightarrow f(x) \in B_{0}
\end{aligned}
$$

Thus, the left side of (2) can be restated as:

$$
\underline{P}=\sum_{j: x \in A_{j} \rightarrow f(x) \in B_{0}} p_{j}
$$

So, since we only know the value of $f(x)$ on $M$ different inputs $x_{k}$, it makes sense to use as an estimator the same expression on the sample data set, that is to define:

$$
\underline{P}_{M}:=\sum_{j: \forall k \leq M, x_{k} \in A_{j} \rightarrow y_{k} \in B_{0}} p_{j}
$$

We will show below that $\underline{P}_{M}$ is, indeed, a good estimator of $\underline{P}$.

Before that, it is useful to consider some of the properties of $\underline{P}_{M}$. First, note that (3) can be restated, by logical expansion of the implication operator and de Morgan over universal quantification, as

$$
\begin{aligned}
\underline{P}_{M} & =\sum_{\forall j: \forall k \leq M, x_{k} \notin A_{j} \vee y_{k} \in B_{0}} p_{j} \\
& =\sum_{\forall j: \nexists k \leq M, x_{k} \in A_{j} \wedge y_{k} \notin B_{0}} p_{j} .
\end{aligned}
$$

In other words, $\underline{P}_{M}$ includes the $p_{j}$ values for any box $A_{j}$ which is not "contradicted" by a data point $\left\langle x_{k}, y_{k}\right\rangle$ such that $x_{k}$ is in the box $A_{j}$, but nonetheless $y_{k} \notin B_{0}$. Note that for $M=0$, there can be no such data points, since $S_{0}$ is empty (there are no data points at all). Thus $\underline{P}_{0}=\sum_{j=1}^{N} p_{j}=1$, so that all boxes $A_{j}$ are included. For $M>0$, as data points $\left\langle x_{k}, y_{k}\right\rangle \in S_{M}$ are encountered for which $y_{k} \notin B_{0}$, all the boxes $A_{j} \ni x_{k}$ become excluded from $\underline{P}_{M}$. Hence $0<M<M^{\prime} \rightarrow \underline{P}_{M} \geq_{M^{\prime}}$, so that $\underline{P}_{M}$ is monotonically non-increasing in $M$.

Consider now a particular box $A_{j}$. There are two possibilities:

1. $\forall x \in A_{j}, f(x) \in B_{0}$ : By $(2), p_{j}$ is not excluded from $\underline{P}$. Moreover, no contradictory data point will be encountered, so $p_{j}$ can never be excluded from $\underline{P}_{M}$.

2. $\exists x \in A_{j}, f(x) \notin B_{0}$ : Now $p_{j}$ will be excluded from $\underline{P}$. But it might be that no such contradictory $x$ is encountered as an $x_{k}$ in $S_{M}$, so that $p_{j}$ may or may not be exluded from $\underline{P}_{M}$. 
Thus we can see that $\forall M>0, \underline{P} \leq \underline{P}_{M}$.

So $\underline{P}_{M}$ is a monotonically non-increasing sequence bounded below by $\underline{P}$. Thus $\underline{P}_{M}$ has a limit, and it's reasonable to ask if that limit is, indeed $\underline{P}$, and to ask about the convergence of $\underline{P}_{M} \longrightarrow \underline{P}$ as $M \longrightarrow \infty$. Our proof actually proves a stronger result: not only does $\underline{P}_{M}$ give the correct value of $\underline{P}$ in the limit as $M \longrightarrow \infty$, but it also does so for a sufficiently large, but finite, number $M_{0}$ of sample points.

Theorem 2 Let $f: \mathbb{R}^{n} \mapsto \mathbb{R}^{m}$ be a continuous function, $S=\left\{\left\langle x_{1}, y_{1}\right\rangle, \ldots,\left\langle x_{k}, y_{k}\right\rangle, \ldots\right\}$ be an infinite sequence of pairs such that the values $x_{k}$ are everywhere dense in $X$, and $y_{k}=f\left(x_{k}\right)$. Let $S_{M}$ be the finite subsequence of $S$ for $k \leq M$, and let $\underline{P}_{M}$ be defined as in (3) on $S_{M}$. Then there exists an integer $M_{0}$ such that $\forall M \geq M_{0}, \underline{P}_{M}=\underline{P}$.

Corollary 3 Given the conditions holding in Theorem 2, then

$$
\lim _{M \longrightarrow \infty} \underline{P}_{M}=\underline{P} .
$$

Proposition 4 The condition that a sequence $\left\{x_{1}, \ldots, x_{k}, \ldots\right\}$ is everywhere dense is satisfied if to select $x_{k}$, we generate independent random vectors - random relative to some probability distribution for which the probability density function $\rho(x)$ is continuous and positive everywhere on the set $X$.

\section{$3.2 \quad$ Upper Probability}

By similar reasoning to $\underline{P}$, according to Theorem 1 , the probability $\bar{P}$ is the sum of the probabilities $p_{j}$ for all boxes $j$ for which $f\left(A_{j}\right) \cap B_{0} \neq \emptyset$. This set theoretical condition can also be expressed in logical terms:

$$
\begin{aligned}
f\left(A_{j}\right) \cap B_{0} \neq \emptyset & \equiv\left\{f(x): x \in A_{j}\right\} \cap B_{0} \neq \emptyset \\
& \equiv \exists x \in \mathbb{R}^{n}, x \in A_{j} \wedge f(x) \in B_{0}
\end{aligned}
$$

Thus, the right side of (2) can be restated as:

$$
\bar{P}=\sum_{j: \exists x \in \mathbb{R}^{n}, x \in A_{j} \wedge f(x) \in B_{0}} p_{j}
$$


So in seeking an estimator for $\bar{P}$, we can similarly advance:

$$
\bar{P}_{M}:=\sum_{j: \exists k \leq M, x_{k} \in A_{j} \wedge y_{k} \in B_{0}} p_{j}
$$

Similarly, but conversely, to $\underline{P}$, we can easily conclude that $\bar{P}_{M}$ is a monotonically non-decreasing sequence bounded above by $\bar{P}$. Thus it also has a limit. Does this limit equal $\bar{P}$ ? Well, unlike $\underline{P}$, this limit may be different from the desired value $\bar{P}$.

Theorem 5 There exists a continuous function $f(x)$ and an everywhere dense sequence $\left\langle x_{k}, y_{k}\right\rangle$ for which $\bar{P}_{M} \rightarrow 0$ and $\bar{P}=1$.

However, a natural minor modification of (2) considered in Sec. 4.2 below will lead to the desired result.

\subsection{Comments}

- (3) and (4) were originally proposed by Joslyn and Helton [9] as estimators of the DempsterShafer uncertainty measures Bel and $\mathrm{Pl}$ respectively.

- As mentioned above, the Dempster-Shafer formalism inspiring this formulation is isomorphic to a random set approach [3]. From this viewpoint, our convergence result can be obtained as particular case of convergence results for random sets [10].

- How algorithmic are (3) and (4)? For each rectangular box $A_{\vec{j}}=\underset{i=1}{\times} A_{j^{(i)}}^{(i)}$, checking whether a given input $x=\left\langle x^{(1)}, \ldots, x^{(i)}, \ldots, x^{(n)}\right\rangle$ belongs to this box means checking that for every $i \in \mathbb{N}_{n}$, the value $x^{(i)}$ belongs to the corresponding interval $A_{j^{(i)}}^{(i)}$ for each $j^{i)} \in \vec{j}$, i.e., checking $\forall i \in \mathbb{N}_{n}, \forall j^{(i)} \in \vec{j}$, whether $\underline{x}_{j(i)}^{(i)} \leq x^{(i)} \leq \bar{x}_{j^{(i)}}^{(i)}$. So since $A_{j}$ and $B_{0}$ are rectangular boxes, checking whether $x_{k} \in A_{j}$ or whether $y_{k} \in B_{0}$ means checking $n$ and $m$ corresponding double inequalities respectively. Thus, computing the above estimates $\underline{P}_{M}$ and $\bar{P}_{M}$ requires finitely many computational steps.

- For the above algorithm, the number of steps is proportional to the total number of boxes and it can actually be quite large. In Sec. 5 we will show how we can decrease the computation time when the number of boxes is large. 
- In the proof of Theorem 2 and Corollary 3, we do not use the fact that $B_{0}$ is a closed box, only that it is a closed set.

\section{Advanced Results}

We now consider some other results which hold for this problem.

\subsection{Continuity and Density Requirements}

Theorem 2 was proven using two assumptions: that the function $f(x)$ is continuous, and that the sequence $x_{k}$ is everywhere dense. The following propositions show that both conditions are necessary.

Theorem 6 There exists a discontinuous function $f(x)$ and an everywhere dense sequence $x_{k}$ for which $\underline{P}_{M} \not \underline{P}$.

The counter-example used in the proof is quite natural. Moreover, for discontinuous functions, not only is it the case that our method cannot extract the correct value $\underline{P}$, but moreover, it is impossible to do so by any method.

Theorem 7 There exists a discontinuous function $f(x)$ and a continuous function $\widetilde{f}(x)$, for which $\underline{P} \neq \underline{\widetilde{P}}$, but for which, for some everywhere dense sequence $x_{k}$, we have $f\left(x_{k}\right)=\widetilde{f}\left(x_{k}\right)$ for all $k$.

Thus, we have the same set of pairs $\left\langle x_{k}, y_{k}\right\rangle$ to start with, so no matter what method we use, we cannot end up with two different values $\underline{P}$.

Similarly, if the sequence is not everywhere dense, we cannot reconstruct $\underline{P}$ no matter what method we use.

Theorem 8 Let the boxes and probabilities be given, and let $B_{0} \subset \mathbb{R}^{m}$ be a closed set. Then, for every sequence $x_{k}$ which is not everywhere dense in $X$, there exist different continuous functions $f(x)$ and $\widetilde{f}(x)$ for which $\underline{P} \neq \underline{\widetilde{P}}$, but for which we have $f\left(x_{k}\right)=\widetilde{f}\left(x_{k}\right)$ for all $k$. 


\subsection{Modification of Upper Probability Conditions}

Similar to the counter-example from the proof of Theorem 6, the counter-example used in the proof of Theorem 5 is also very natural. So, we have to modify the original estimator $\bar{P}_{M}$ for the upper probability. Fortunately, such a modification is relatively easy and straightforward, introducing only modest considerations for the semantics of the kinds of risk and reliability problems concerning us.

First, we know that the box of desirable states $B_{0}$ can be characterized by intervals on the dimensions of the output space as

$$
B_{0}=\underset{l=1}{\stackrel{m}{\times}} B_{0}^{(l)}=\underset{l=1}{\times}\left[\underline{y}_{0}^{(l)}, \bar{y}_{0}^{(l)}\right] .
$$

So now consider a small positive real number $\alpha>0$, and define the extended desirable box as:

$$
B_{\alpha}:=\left[\underline{y}_{0}^{(1)}-\alpha, \bar{y}_{0}^{(1)}+\alpha\right] \times\left[\underline{y}_{0}^{(2)}-\alpha, \bar{y}_{0}^{(2)}+\alpha\right] \times \ldots \times\left[\underline{y}_{0}^{(m)}-\alpha, \bar{y}_{0}^{(m)}+\alpha\right] .
$$

Elements of this extended box $B_{\alpha}$ do not necessarily satisfy all $2 m$ desired inequalities generated by the outpute intervals $B_{0}^{(l)}$, but their deviation from each of these inequalities does not exceed $\alpha$. Our reliability requirement now becomes that $f(x)$ belongs to this extended box: $f(x) \in B_{\alpha}$. The upper bound for this probability is then

$$
\bar{P}_{\alpha}=\sum_{j: f\left(A_{j}\right) \cap B_{\alpha} \neq \emptyset} p_{j}
$$

Since the modified condition $f(x) \in B_{\alpha}$ is less demanding than the original condition $f(x) \in B_{0}$, it is now easier for a state to be desirable, so the probability for a state to be desirable is higher: $\bar{P} \leq \bar{P}_{\alpha}$. The actual upper probability can be anywhere between $\bar{P}$ and $\bar{P}_{\alpha}$. From this viewpoint, when we compute an estimate for $\bar{P}$, it is also reasonable, instead of sticking to the original set $B_{0}$, to depart from (4) and instead use our slightly enlarged set $B_{\alpha}$ :

$$
\bar{P}_{\alpha, M}:=\sum_{j: \exists k \leq M, x_{k} \in A_{j} \wedge y_{k} \in B_{\alpha}} p_{j}
$$

Theorem 9 Let $f: \mathbb{R}^{n} \mapsto \mathbb{R}^{m}$ be a continuous function, $S=\left\langle x_{k}, y_{k}\right\rangle, k>0$ be an infinite sequence of pairs such that the values $x_{k}$ are everywhere dense in $X$, and $y_{k}=f\left(x_{k}\right)$. Let $S_{M}$ be the finite subsequence of $S$ for $k \leq M$, and for a small number $\alpha>0$, let $\bar{P}_{\alpha, M}$ be defined as in (3) on $S_{M}$. Then there exists an integer $M_{0}$ such that $\forall M \geq M_{0}, \bar{P}_{\alpha, M} \in\left[\bar{P}, \bar{P}_{\alpha}\right]$.

The main idea of the above result is that the required bounds on the state variables $y_{k}$ are not exact, they can be exceeded a little bit - by some small value $\alpha$ - without any harm to the system. 
In the above result, we used the same value $\alpha>0$ for every $M$. Intuitively, the more pairs we have, the more accurately we can describe the requirements. It is therefore seems reasonable, instead of selecting a single $\alpha$ for all $M$, to make $\alpha$ decrease to 0 when $M \rightarrow \infty: \alpha_{M} \rightarrow 0$. Then, if we can still prove the inequality $\bar{P} \leq \bar{P}_{\alpha_{M}, M} \leq \bar{P}_{\alpha_{M}}$, we will be able to conclude, in the limit $M \rightarrow \infty$, that $\bar{P}_{\alpha_{M}, M} \rightarrow \bar{P}$. Alas, as we will show, this is not possible - and in this sense Theorem 9 is the best we can get.

Theorem 10 Let $\alpha_{M} \rightarrow 0$ be a sequence of positive real numbers. Then, there exists a continuous function $f(x)$ and an infinite sequence of pairs $\left\langle x_{k}, y_{k}\right\rangle$ for which the values $x_{k}$ are everywhere dense in $X$, and $\bar{P}_{\alpha_{M}, M} \nrightarrow \bar{P}$.

\section{Estimator Calculations for Large Numbers of Inputs}

Equations (3), (4), and (5) require that we analyze every single box $j$. If for each variable $x^{(i)}$, we have $N^{(i)}$ possible intervals, then have $N=\prod_{i=1}^{N} N^{(i)}$ boxes. In a nuclear facility example, we could have about $n=100$ variables, and at least two boxes $N^{(i)} \geq 2$ for each variable. Thus, the total number of boxes is $2^{100}$ - which is approximately $10^{30}$. Testing that many boxes is well beyond the capacity of modern computers. So what do we do?

A natural idea is to use Monte-Carlo simulation to estimate, e.g., $\underline{P}_{M}$. Indeed, (3) can be interpreted as follows. For each box $j$, let

$$
\chi_{M}(j)=\left\{\begin{array}{ll}
1, & \forall k \leq M, x_{k} \in A_{j} \rightarrow y_{k} \in B_{0} \\
0, & \text { otherwise }
\end{array} .\right.
$$

Then (3) takes the form $\underline{P}=\sum_{j=1}^{N} p_{j} \chi_{M}(j)$. In other words, $\underline{P}$ is a mathematical expectation of $\chi_{M}(j)$ under the probability distribution in which each box $A_{j}$ appears with probability $p_{j}$.

Recall that we constructed a bijective mapping between the integers $\mathbb{N}_{N}$ and the vectors $\vec{j}=$ $\left\langle j^{(1)}, \ldots, j^{(n)}\right\rangle$. Since the probability $p_{j}$ is defined as the product $\prod_{i=1}^{N} p_{j^{(i)}}^{(i)}$ of the corresponding probabilities $p_{j(i)}^{(i)}$, to get this probability distribution, it is sufficient to independently select each situation $j^{(i)} \in \vec{j}$ with probability $p_{j^{(i)}}^{(i)}$. This can be done, e.g., as follows: we subdivide the interval $[0,1]$ into $N^{(i)}$ subintervals of lengths $p_{1}^{(i)}, p_{2}^{(i)}$, etc., - i.e., into the intervals $\left[0, p_{1}^{(i)}\right],\left[p_{1}^{(i)}, p_{1}^{(i)}+p_{2}^{(i)}\right]$, etc., and then run a random number generator corresponding to the uniform distribution on $[0,1]$ to select a situation depending on into which interval the resulting random number falls. 
Before we show the algorithm, we introduce some auxiliary computations:

- For each $i \in \mathbb{N}_{n}$, we compute the values $q_{0}^{(i)}:=0$ and $q_{j^{(i)}+1}^{(i)}:=q_{j^{(i)}}^{(i)}+p_{j^{(i)}+1}^{(i)}, 0 \leq j^{(i)} \leq$ $N^{(i)}-1$.

- For each $k \in \mathbb{N}_{M}$, we check whether $y_{k} \in B_{0}$, i.e., whether the corresponding inequalities $\underline{y}^{(l)} \leq y_{k}^{(l)} \leq \bar{y}^{(l)}$ are satisfied for all $l \in \mathbb{N}_{m}$.

Then, to estimate $\underline{P}$, we select the number of runs $R$; the larger $R$ is, the better the estimate.

Now for the algorithm itself. For each run $r \in \mathbb{N}_{R}$, we do the following:

- For $i$ from 1 to $n$ :

- Run a random number generator (RNG) corresponding to the uniform distribution on the interval $[0,1]$ and store the result in $r^{(i)}$.

- By comparing the result $r^{(i)}$ of this RNG with the values $q_{j^{(i)}}^{(i)}$, we find and retain the value $j^{(i)}$ for which $r^{(i)} \in\left[q_{j^{(i)}-1}^{(i)}, q_{j^{(i)}}^{(i)}\right]$.

- For every $k \in \mathbb{N}_{M}$ for which $y_{k} \notin B_{0}$, we check whether $x_{k} \in A_{j}$, i.e., whether the inequalities $\underline{x}_{j(i)}^{(i)} \leq x_{k}^{(i)} \leq \bar{x}_{j(i)}^{(i)}$ hold for all $i \in \mathbb{N}_{n}$. After that:

- If for some $k$ for which $y_{k} \notin B_{0}$, we have $x_{k} \in A_{j}$, this means that

$$
\neg\left(\forall k \leq M, x_{k} \in A_{j} \rightarrow y_{k} \in B_{0}\right),
$$

so we set $\chi_{r}=0$;

- Otherwise, we set $\chi_{r}=1$.

Finally, we take the average $\sum_{r=1}^{R} \chi_{r} / R$ as the desired estimate for $\underline{P}_{M}$.

The above algorithm is a standard Monte-Carlo algorithm, so when $R \rightarrow \infty$, its result converges to $\underline{P}_{M}$. Due Theorem 2, for sufficiently large $M$, we have $\underline{P}_{M}=\underline{P}$. Therefore, we can conclude that for sufficiently large $M$, the results of the above algorithm converge to $\underline{P}_{M}$.

An algorithm for computing $\bar{P}_{\alpha, M}$ is similar to the algorithm for computing $\underline{P}_{M}$, with the only difference that here:

- $\chi_{M}(j)=1$ if $\exists k \leq M, x_{k} \in A_{j} \wedge y_{k} \in B_{\alpha}$, and

- $\chi_{M}(j)=0$ otherwise. 
Interval Probabilistic Approach

\section{A Theorem 1}

We need to show three things:

1. For every consistent probability distribution $P, \underline{P} \leq \operatorname{Prob}\left(f(x) \in B_{0}\right) \leq \bar{P}$.

By definition, the fact that the probability measure $P(A)$ is consistent means that

$$
P(A)=\sum_{j=1}^{N} p_{j} P_{j}(A)
$$

for some probability measures $P_{j}(A)$ which are concentrated within the corresponding boxes $A_{j}$. The probability $\operatorname{Pr}\left(f(x) \in B_{0}\right)$ is equal to the probability that $x$ belongs to the set $f^{-1}\left(B_{0}\right)$ of all values $x$ for which $f(x) \in B_{0}$, so that $\operatorname{Pr}\left(f(x) \in B_{0}\right)=P\left(f^{-1}\left(B_{0}\right)\right)$. In particular, for $A=f^{-1}\left(B_{0}\right)$, we have $P:=P\left(f^{-1}\left(B_{0}\right)\right)=\sum_{j=1}^{N} p_{j} P_{j}\left(f^{-1}\left(B_{0}\right)\right)$.

For each combined situation $j$, the probability measure $P_{j}(A)$ is located on the box $A_{j}$. Thus:

- If a box $A_{j}$ has no elements $x$ with $f(x) \in B_{0}$ - i.e., if $f\left(A_{j}\right)$ has no common elements with $B_{0}$ - we have $P_{j}\left(f^{-1}\left(B_{0}\right)\right)=0$.

- For those boxes that do contain elements $x$ with $f(x) \in B_{0}$ - i.e., for which $f\left(A_{j}\right)$ has a non-empty intersection with $B_{0}$, the conditional probability $P_{j}\left(f^{-1}\left(B_{0}\right)\right)$ - just like any other probability - cannot exceed 1 .

Thus, replacing $P_{j}\left(f^{-1}\left(B_{0}\right)\right)$ with 0 for boxes for which $f\left(A_{j}\right)$ that do not intersect $B_{0}$ and with 1 for boxes that do, we get an upper bound for $P$ - the upper bound which is exactly the expression $\bar{P}$ from (2).

Similarly, since the probability measure $P_{j}(A)$ is located on the box $A_{j}$, we have $P_{j}\left(A_{j}\right)=1$. Therefore:

- $P_{j}\left(f^{-1}\left(B_{0}\right)\right)=1$ for all boxes $A_{j}$ for which $f\left(B_{0}\right) \subseteq A_{j}$.

- For all other boxes, the conditional probability is a non-negative number $P_{j}\left(f^{-1}\left(B_{0}\right)\right) \geq$ 0 - just like any other probability.

Thus, replacing $P_{j}\left(f^{-1}\left(B_{0}\right)\right)$ with 0 for boxes for which $f\left(A_{j}\right)$ does not contain $B_{0}$ and with 1 for boxes that do, we get a lower bound for $P$ - the lower bound which is exactly the expression $\underline{P}$ from (2). 
2. There exists a consistent probability distribution $P(A)$ for which $\operatorname{Pr}\left(f(x) \in B_{0}\right)=\underline{P}$.

We select the probability $P_{j}(A)$ in one of two ways:

$f\left(A_{j}\right) \subseteq B_{0}$ : Select an arbitrary point $x_{j} \in A_{j}$, and select $P_{j}(A)$ such that this point occurs with probability 1, i.e., for which $P_{j}(A)=1$ if $x_{j} \in A$ and $P_{j}(A)=0$ otherwise.

$f\left(A_{j}\right) \nsubseteq B_{0}$ : By definition of the subset relation, there exists a point $y_{j} \in f\left(A_{j}\right)$ with $y_{j} \notin B_{0}$. Since $y_{j} \in f\left(A_{j}\right)$, there exists a value $x_{j} \in A_{j}$ for which $y_{j}=f\left(x_{j}\right)$ and for which, therefore, $f\left(x_{j}\right) \notin B_{0}$. For each such box, as $P_{j}(A)$, we take a probability distribution in which this point occurs with probability 1, i.e., for which $P_{j}(A)=1$ if $x_{j} \in A$ and $P_{j}(A)=0$ otherwise.

For these selected distributions, $P_{j}\left(f^{-1}\left(B_{0}\right)\right)=1$ for boxes for which $f\left(A_{j}\right) \subseteq B_{0}$, and $P_{j}\left(f^{-1}\left(B_{0}\right)\right)=0$ for all other boxes. Thus, for the resulting distribution $P(A)$, the probability $P=P\left(f^{-1}\left(B_{0}\right)\right)$ is equal to $\underline{P}$.

3. There exists a consistent probability distribution $P(A)$ for which $\operatorname{Pr}\left(f(x) \in B_{0}\right)=\bar{P}$.

- For each box $A_{j}$ for which $f\left(A_{j}\right) \cap B_{0}=\emptyset$, we select an arbitrary point $x_{j}$ within this box, and, as $P_{j}(A)$, take a probability distribution in which this point occurs with probability 1, i.e., for which $P_{j}(A)=1$ if $x_{j} \in A$ and $P_{j}(A)=0$ otherwise.

- For each box $A_{j}$ for which $f\left(A_{j}\right) \cap B_{0} \neq \emptyset$, by definition of a non-empty set, there exists a point $y_{j} \in f\left(A_{j}\right) \cap B_{0}$. Since $y_{j} \in f\left(A_{j}\right)$, there exists a value $x_{j} \in A_{j}$ for which $y_{j}=f\left(x_{j}\right)$ and for which, therefore, $y_{j}=f\left(x_{j}\right) \in B_{0}$. For each such box, as $P_{j}(A)$, we take a probability distribution in which this point occurs with probability 1, i.e., for which $P_{j}(A)=1$ if $x_{j} \in E$ and $P_{j}(A)=0$ otherwise.

For thus selected distributions, $P_{j}\left(f^{-1}\left(B_{0}\right)\right)=0$ for boxes for which $f\left(A_{j}\right) \cap B_{0}=\emptyset$, and $P_{j}\left(f^{-1}\left(B_{0}\right)\right)=1$ for all other boxes. Thus, for the resulting distribution $P(A)$, the probability $P=P\left(f^{-1}\left(B_{0}\right)\right)$ is equal to $\bar{P}$.

\section{B Theorem 2}

We will show that for every box $A_{j}$, there exists an integer $M_{j}$ such that for every $M \geq M_{j}$, the condition $f\left(A_{j}\right) \subseteq B_{0}$ is equivalent to $\forall k \leq M, x_{k} \in A_{j} \rightarrow y_{k} \in B_{0}$. Then, if we take the largest of 
theses values $M_{j}$ as $M_{0}$, we will be able to conclude that for every $M \geq M_{0}$, these two conditions are equivalent to each other for every box $j$. Thus, by comparing the formula for $\underline{P}$ from (2) and the definition of $\underline{P}_{M}$ from (3), we will be able to conclude that indeed $\underline{P}_{M}=\underline{P}$.

Let us show that the two conditions are indeed equivalent. The equivalence proof will be different for two cases: when $f\left(A_{j}\right) \subseteq B_{0}$ and when $f\left(A_{j}\right) \nsubseteq B_{0}$. Specifically, we will show that:

- When $f\left(A_{j}\right) \subseteq B_{0}$, then the finite analog of this condition is also satisfied, i.e., $\forall k \leq M, x_{k} \in$ $A_{j} \rightarrow y_{k} \in B_{0}$, and

- When $f\left(A_{j}\right) \nsubseteq B_{0}$, then the finite analog of this condition is also satisfied, i.e., $\neg(\forall k \leq$ $M, x_{k} \in A_{j} \rightarrow y_{k} \in B_{0}$, or, equivalently,

$$
\exists k \leq M, x_{k} \in A_{j} \wedge y_{k} \notin B_{0} .
$$

$f\left(A_{j}\right) \subseteq B_{0}$ : For every $x \in A_{j}$, we have $f(x) \in B_{0}$. In particular, when $x=x_{k}$, from $x_{k} \in A_{j}$, we will thus be able to conclude that $y_{k}=f\left(x_{k}\right) \in B_{0}$, so the the finite version of this condition is also satisfied.

$f\left(A_{j}\right) \not B_{0}$ : So there exists a point $x^{*} \in A_{j}$ for which $y^{*}:=f\left(x^{*}\right) \notin B_{0}$ - or, equivalently, $f\left(x^{*}\right) \in \overline{B_{0}}$, where - denotes set complement. Since $B_{0}$ is a closed set, its complement $\overline{B_{0}}$ is an open set. Therefore, together with a point $f\left(x^{*}\right)$, it contains an entire open ball $B_{\varepsilon}\left(f\left(x^{*}\right)\right):=\left\{y: d\left(y, y^{*}\right)<\varepsilon\right\}$ of a positive radius $\varepsilon>0$ with a center in $f\left(x^{*}\right)$. So, if $d\left(y, f\left(x^{*}\right)\right)<\varepsilon$, then $y \in \overline{B_{0}}$, i.e., $y \notin B_{0}$.

Since the function $f(x)$ is continuous, in particular, it is continuous at the point $x=x^{*}$. By definition of continuity, this means that for every $\varepsilon>0$, there exists a $\delta>0$ such that if $d\left(x, x^{*}\right)<\delta$, then $d\left(f(x), f\left(x^{*}\right)\right)<\varepsilon$. We already know that $d\left(f(x), f\left(x^{*}\right)\right)<\varepsilon$ means that $f(x) \in \overline{B_{0}}$, i.e., $f(x) \notin B_{0}$. Therefore, we can conclude that when $d\left(x, x^{*}\right)<\delta$, then $f(x) \notin B_{0}$.

We know that the point $x^{*}$ belongs to the box $A_{j}$. There are two possibilities:

$x^{*}$ is strictly inside $A_{j}$ : Let $\delta_{0}$ be the smallest distance from $x^{*}$ to any of the edges. Then, $d\left(x, x^{*}\right)<\delta_{0}$ implies that $x$ is also inside the box $A_{j}$. Hence, if we take $\delta_{1}:=\min \left(\delta, \delta_{0}\right)$, we can conclude the following: when $d\left(x, x^{*}\right)<\delta_{1}$, then $x \in A_{j}$ and $f(x) \notin B_{0}$. 
Interval Probabilistic Approach

We assumed that the sequence $\left\{x_{k}\right\}$ is everywhere dense in $X$. By definition, this means that for every point $x \in X$ (in particular, for $x^{*} \in A_{j}$ ) and for every $\delta>0$ (in particular, for $\left.\delta=\delta_{1}\right)$, there exists a point $x_{k^{*}}$ for which $d\left(x_{k^{*}}, x^{*}\right)<\delta_{1}$. We already know that in this case, $x_{k^{*}} \in A_{j}$ and $y_{k}^{*}=f\left(x_{k^{*}}\right) \notin B_{0}$. Therefore, for every $M \geq k^{*}$, there exists a $k \leq M$ (namely, $k=k^{*}$ ) for which $x_{k} \in A_{j} \wedge y_{k} \notin B_{0}$. Hence, if we take this $k^{*}$ as $M_{j}$, then the finite analog of the condition $f\left(A_{j}\right) \nsubseteq B_{0}$ is indeed satisfied for all $M \geq M_{j}$.

$x^{*}$ is on the border of $A_{j}$ : For each point on the border and for every $\delta$, there exists a $\delta$-close point inside the box. So, there exists a point $x^{* *}$ inside the box $A_{j}$ for which $d\left(x^{*}, x^{* *}\right)<\delta$ - and therefore, $f\left(x^{* *}\right) \notin B_{0}$. So, we have an internal point $x^{* *} \in A_{j}$ for which $f\left(x^{* *}\right) \notin B_{0}$. For this new point, we can repeat the same proof that we had starting with $x^{*}$, and conclude that there exists an $M_{j}$ such that for every $M \geq M_{j}$, there exists a $k \leq M$ for which $x_{k} \in A_{j} \wedge y_{k} \notin B_{0}$ - i.e., the finite analog of the condition $f\left(A_{j}\right) \nsubseteq B_{0}$ is indeed satisfied for all $M \geq M_{j}$.

\section{Corollary 3}

Follows immediately.

\section{Proposition 4}

Let $x \in \bigcup_{j=1}^{N} A_{j}$ be a point, and $\varepsilon>0$. Let us show that with probability 1 , one of the points $x_{k}$ will be $\varepsilon$-close to $x$. Indeed, let $I:=X \cap B_{\varepsilon}(x)$, where $B_{\varepsilon}(x)$ is the open ball around $x$. For the probability distribution $P_{\text {sel }}$ that we use to select the points $x_{k}$, the probability $P_{\text {sel }}(B)$ that a randomly picked vector $x$ is inside $B$ is equal to $P_{\text {sel }}(B)=\int_{B} \rho(y) \mathrm{d} y$. The intersection $B$ has a positive volume, so, since the probability density function $\rho(y)$ is positive and continuous, this integral $P_{\text {sel }}(B)$ is also positive.

Hence, for every $k$, the probability that $x_{k} \notin B$ is equal to $1-P_{\text {sel }}(B)<1$. Since we assume that the points are independently selected on each iteration, the probability that on each of $M$ selections, we get a point $x_{k} \notin B$ is equal to the product of the corresponding $M$ probabilities, i.e., to $\left(1-P_{\text {sel }}(B)\right)^{M}$. When $M \rightarrow \infty$, this probability tends to 0 , so we conclude that the probability that none of the infinitely many points $x_{k}$ is inside $B$ is equal to 0 . So, with probability 1 , there is 
a point $x_{k}$ inside $B$ - i.e., a point $x_{k} \in X$ for which $d\left(x, x_{k}\right) \leq \varepsilon$.

Since every ball contains a smaller ball with rational center and rational radius, it is sufficient to show that we can find $x_{k}$ within each ball of rational center and radius. There are countably many such balls, and for each, the probability of not having $x_{k}$ inside it is 0 . Thus, the probability that one of these balls does not contain any of $x_{k}$ 's is also 1 - so with probability 1 , every ball has a point from $x_{k}$, which means, by definition, that the sequence $\left\{x_{k}\right\}$ is everywhere dense.

\section{E Theorem 5}

Let $n=1, N^{(1)}=1$, and $A_{1}^{(1)}=[0,1]\left(\right.$ with $\left.p_{1}^{(1)}=1\right)$. Let $x_{k}$ be an arbitrary everywhere dense sequence of numbers from the open interval $(0,1)$ - e.g., a sequence obtained by using a random number generator that generates numbers uniformly distributed on the interval $[0,1]$.

Let us take $f(x)=x$ and $B_{0}=[1,2]$. Then, $f\left(A_{j}\right) \cap B_{0} \neq \emptyset$, so $\bar{P}=1$. On the other hand, since the values $x_{k}$ are taken from the inside of the interval $[0,1]$, none of these values is equal to 1 , and therefore, none of the points $x_{k} \in A_{j}$ has the property that $y_{k} \in B_{0}$. So, for every $M$, we have $\bar{P}_{M}=0$.

\section{F $\quad$ Theorem 6}

Again, let $n=1, N^{(1)}=1$, and $A_{1}^{(1)}=[0,1]\left(\right.$ with $p_{1}^{(1)}=1$ ). Let $x_{k}$ be an arbitrary everywhere dense sequence of positive numbers - e.g., a sequence obtained by using a random number generator that generates numbers uniformly distributed on the interval $[0,1]$.

Let $f(x)$ be the sign function

$$
f(x):= \begin{cases}0, & x=0 \\ 1, & x>0 \\ -1, & x<0\end{cases}
$$

and let $B_{0}=[0.5,1.5]$. Then, for the only box $A_{j}=[0,1]$, we have $f\left(A_{j}\right)=\{0,1\} \nsubseteq B_{0}$, so $\underline{P}=0$. However, since all the values $x_{k}$ are positive, we have $y_{k}=f\left(x_{k}\right)=1$ hence $y_{k} \in B_{0}$; thence $\underline{P}_{M}=1$ for all $M$. Here, as $M \rightarrow \infty$, we have $\underline{P}_{M} \rightarrow 1$, so $\underline{P}_{M} \nrightarrow \underline{P}$. 


\section{G $\quad$ Theorem 7}

Let us take $f(x)$, the boxes, and the everywhere dense sequence the same as in the proof of Theorem 6 , and $\widetilde{f}(x)=1$ for all $x$. Then, $0=\underline{P} \neq \underline{\widetilde{P}}=1$.

\section{H Theorem 8}

Since the sequence $x_{k}$ is not everywhere dense, there exists a ball $\beta_{r}\left(x^{*}\right) \subseteq \mathbb{R}^{n}$ that is not covered by any element from this sequence. Since $B_{0} \subset \mathbb{R}^{m}$, there exists a point $y^{*} \in \mathbb{R}^{m}, y^{*} \notin B_{0}$. Define the function $f(x)$ as a constant equal to some point $y^{* *} \in B_{0}$; for this function, $P\left(f^{-1}\left(B_{0}\right)\right)=1$. As $\widetilde{f}(x)$, we take a function

$$
\widetilde{f}(x)=y^{* *}+\max \left(0,1-\frac{d\left(x, x^{*}\right)}{r}\right) \cdot\left(y^{*}-y^{* *}\right) .
$$

One can easily see that $\tilde{f}(x)=f(x)$ for all $x \notin \beta_{r}\left(x^{*}\right)$, so the sequences $\left\langle x_{k}, y_{k}\right\rangle$ for these two functions are indeed the same. However, since $\tilde{f}\left(x^{*}\right)=y^{*} \notin B_{0}$, there exists a box $A_{j}$ - namely, any box that contains the point $x^{*}$ - for which $f\left(A_{j}\right) \nsubseteq B_{0}$ and therefore, we have $\underline{\widetilde{P}}<1$ (while $\underline{P}=1)$.

\section{Theorem 9}

We have already proven, in the discussion of the formula for $\bar{P}_{M}$, that $\bar{P}_{M} \leq \bar{P}$. Thus, we also have $\bar{P}_{\alpha, M} \leq \bar{P}_{\alpha}$. So, to complete our proof, we must show that there exists an $M_{0}$ such that for every $M \geq M_{0}$, we have $\bar{P} \leq \bar{P}_{\alpha, M}$.

Similarly to the proof of Theorem 2, we will prove that for every box $A_{j}$, there exists a value $M_{j}$ such that for every $M \geq M_{j}$, the condition $f\left(A_{j}\right) \cap B_{0} \neq \emptyset$ implies that

$$
\exists k \leq M, x_{k} \in A_{j} \wedge y_{k} \in B_{\alpha} .
$$

If we prove this, then, for every $M \geq M_{0}:=\max _{j} M_{j}$, we will be able to conclude that all the terms $p_{j}$ involved in the formula for $\bar{P}$ are also included in the sum that defines $\bar{P}_{\alpha, M}$ and therefore, that indeed $\bar{P} \leq \bar{P}_{\alpha, M}$.

Indeed, let $f\left(A_{j}\right) \cap B_{0} \neq \emptyset$. This means that there exists a value $y^{*} \in B_{0}$ for which $y^{*} \in f\left(A_{j}\right)$, i.e., for which $y^{*}=f\left(x^{*}\right)$ for some $x^{*} \in A_{j}$. 
Since the function $f(x)$ is continuous, in particular, it is continuous at the point $x=x^{*}$. By definition of continuity, this means that for every $\alpha>0$, there exists a $\delta>0$ such that if $d\left(x, x^{*}\right)<\delta$, then $d\left(f(x), f\left(x^{*}\right)\right)<\alpha / 2$. We already know that $y^{*}=f\left(x^{*}\right) \in B_{0}$, hence $f(x)$ belongs to $B_{\alpha}$ (actually, it even belongs to $\left.B_{\alpha / 2}\right)$. Therefore, we can conclude that when $d\left(x, x^{*}\right)<\delta$, then $f(x) \in B_{\alpha}$.

We know that the point $x^{*}$ belongs to the box $A_{j}$. There are two possibilities:

$x^{*}$ is inside $A_{j}$ : Let $\delta_{0}$ be the smallest distance from $x^{*}$ to any of the edges. Then, $d\left(x, x^{*}\right)<\delta_{0}$ implies that $x$ is also inside the box $A_{j}$. Hence, if we take $\delta_{1}:=\min \left(\delta, \delta_{0}\right)$, we can conclude that when $d\left(x, x^{*}\right)<\delta_{1}$, then $x \in A_{j}$ and $f(x) \in B_{\alpha}$.

We assumed that the sequence $\left\{x_{k}\right\}$ is everywhere dense in $X$. By definition, this means that for every point $x \in X$ (in particular, for $x^{*} \in A_{j}$ ) and for every $\delta>0$ (in particular, for $\left.\delta=\delta_{1}\right)$, there exists a point $x_{k^{*}}$ for which $d\left(x_{k^{*}}, x^{*}\right)<\delta_{1}$. We already know that in this case, $x_{k^{*}} \in A_{j}$ and $y_{k^{*}}=f\left(x_{k^{*}}\right) \in B_{\alpha}$. Therefore, for every $M \geq k^{*}$, there exists a $k \leq M$ (namely, $k=k^{*}$ ) for which $x_{k} \in A_{j} \wedge y_{k} \in B_{\alpha}$. Hence, if we take this $k^{*}$ as $M_{j}$, then the finite analog of the condition $f\left(A_{j}\right) \cap B_{\alpha} \neq \emptyset$ is indeed satisfied for all $M \geq M_{j}$.

$x^{*}$ is on the border of $A_{j}$ : For each point on the border and for every $\delta$, there exists a $\delta$-close point inside the box. So, there exists a point $x^{* *}$ inside the box $A_{j}$ for which $d\left(x^{*}, x^{* *}\right)<\delta-$ and therefore, $f\left(x^{* *}\right) \in B_{\alpha / 2}$. So, we have an internal point $x^{* *} \in A_{j}$ for which $f\left(x^{* *}\right) \in B_{\alpha / 2}$. For this new point, we can repeat the same proof that we had starting with $x^{*}$, and conclude that there exists an $M_{j}$ such that for every $M \geq M_{j}$, there exists a $k \leq M$ for which $x_{k} \in A_{j} \wedge y_{k} \in B_{\alpha}$ - i.e., the finite analog of the condition $f\left(A_{j}\right) \cap B_{\alpha} \neq \emptyset$ is indeed satisfied for all $M \geq M_{j}$.

\section{J Theorem 10}

Without losing generality, we can assume that $\alpha_{M}<1$ for all $M$.

In this proof, we will use the same box and the same continuous function that was used in the proof of Theorem 5 that $\bar{P}_{M} \nrightarrow \bar{P}$ : namely, we take $n=1, N^{(1)}=1, A_{1}^{(1)}=[0,1]\left(\right.$ with $\left.p_{1}^{(1)}=1\right)$, $f(x)=x$, and $B_{0}=[1,2]$. For this choice, $\bar{P}=1$. 
We will show that for an appropriately chosen everywhere dense sequence $\left\langle x_{k}, f\left(x_{k}\right)\right\rangle$, we will have $\bar{P}_{\alpha_{M}, M}=0$ for all $M-$ and thus, $\bar{P}_{\alpha_{M}, M} \nrightarrow \bar{P}$.

For this, we must make sure that for every $k \leq M$, we have $y_{k} \notin B_{\alpha_{M}}$. For our choice of $B_{0}=[1,2]$, we have $B_{\alpha}=[1-\alpha, 2+\alpha]$. For our choice of $f(x)=x$, we have $y_{k}=x_{k}$. Thus, the condition that we need to satisfy is $x_{k}<1-\alpha_{M}$ for all $M \geq k$. In the limit $M \rightarrow \infty, \alpha_{M} \rightarrow 0$, so this condition is satisfied - provided, of course, that $x_{k}<1$. The requirement that $x_{k}$ is smaller than all possible values $1-\alpha_{k}, 1-\alpha_{k+1}, \ldots$, is equivalent to requiring that $x_{k}$ is smaller than the smallest of the values $1-\alpha_{k}, 1-\alpha_{k+1}, \ldots$. The difference between 1 and a number is the smallest when the subtracted number is the largest, therefore, the above condition is equivalent to $x_{k}<1-\beta_{k}$, where $\beta_{k}:=\max \left(\alpha_{k}, \alpha_{k+1}, \ldots\right)$.

Since $\alpha_{M} \rightarrow 0$, we can conclude that $\beta_{k} \rightarrow 0$, and one can easily see that $\beta_{k}$ is a monotonic sequence: $\beta_{k} \geq \beta_{k+1} \geq \ldots$ So, to complete the proof, it is sufficient to find an everywhere dense sequence $x_{k}$ of numbers from the interval $[0,1]$ for which $x_{k}<1-\beta_{k}$ for some given monotonic sequence $\beta_{k} \rightarrow 0$.

Since $\alpha_{M}<1$ for all $M$, we can conclude that $\beta_{k}=\max \left(\alpha_{k}, \alpha_{k+1}, \ldots\right)<1$ for all $k$.

To obtain such a sequence $x_{k}$, let us start with an arbitrary everywhere dense sequence $y_{1}, y_{2}, \ldots$ of numbers from the open interval $(0,1)$. Let us denote $y_{0}:=0$. Based on this sequence, we will design a new everywhere dense sequence $x_{k}$; this new sequence will consist of zero, one, or several repetitions of $y_{0}=0$, followed by one or several repetitions of $y_{1}$, then one or several repetitions of $y_{2}$, etc. Since all the elements from $y_{k}$ are also in the sequence $x_{k}$, this new sequence is also everywhere dense in the interval $[0,1]$.

We start by checking whether $y_{1}<1-\beta_{1}$.

- If this inequality is satisfied, we start repeating $y_{1}$, i.e., take $x_{1}=y_{1}$.

- If this inequality is not satisfied, we take $x_{1}=y_{0}=0$.

In both cases, we have $x_{1}<1-\beta_{1}$ :

- In the first case, it is true due to our choice of $x_{1}$.

- In the second case, since $\beta_{k}<1$, we have $0<1-\beta_{1}$. 
In general, if we have already selected $x_{1}, \ldots, x_{k}$, and $x_{k}=y_{l}$ for some $l$, then, to select $x_{k+1}$, we check whether $y_{l+1}<1-\beta_{k+1}$ :

- If this inequality is satisfied, we start repeating $y_{l+1}$, i.e., take $x_{k+1}=y_{l+1}$.

- If this inequality is not satisfied, we continue to take $x_{k+1}=y_{l}$.

In both cases, we have $x_{k+1}<1-\beta_{k+1}$ :

- In the first case, it is true due to our choice of $x_{k+1}$.

- In the second case, since we had $x_{k}=y_{l}<1-\beta_{k}$ and $\beta_{k}$ is a monotonic sequence $\beta_{k} \geq \beta_{k+1}$, we conclude that $x_{k+1}=y_{l}<1-\beta_{k} \leq 1-\beta_{k+1}$, i.e., that $x_{k+1}<1-\beta_{k+1}$.

To complete the proof, we must show that the process of selecting $x_{k}$ will not indefinitely stumble on a value $y_{l}$ and that eventually, it will move on to the next value - thus guaranteeing that all values $y_{l}$ will be covered. Indeed, the value $y_{l}$ is selected as $x_{k}$ only until the inequality $y_{l+1}<1-\beta_{k}$ is not satisfied, i.e., until we have $y_{l+1} \geq 1-\beta_{k}$. This cannot be true for arbitrarily large $k$ because then, in the limit $k \rightarrow \infty$, we would have $y_{l+1} \geq 1$, and we assumed that all the values $y_{k}$ are from the open interval $(0,1)$. So, all the values $y_{l}$ are indeed covered.

\section{Acknowledgments}

This work was partly supported by a research grant from Sandia National Laboratories as part of the Department of Energy Accelerated Strategic Computing Initiative (ASCI), by NASA under cooperative agreement NCC5-209 and grant NCC2-1232, by the Future Aerospace Science and Technology Program (FAST) Center for Structural Integrity of Aerospace Systems, effort sponsored by the Air Force Office of Scientific Research, Air Force Materiel Command, USAF, under grant F49620-00-1-0365, and by NSF grants CDA-9522207, EAR-0112968, EAR-0225670, and 9710940 Mexico/Conacyt.

\section{References}

[1] Dempster, AP: (1968) "Upper and Lower Probabilities Generated by a Random Interval", Annals of Mathematical Statistics, v. 39:3, pp. 957-966 
[2] Ferson, Scott; Joslyn, CA; and Helton, JC; Oberkampf, WL; and Sentz, K: (2004) "Summary of the Epistemic Uncertainty Workshop: Consensus Amid Diversity", Reliability Engineering and Systems Safety, v. 95:1-3, pp. 355-370

[3] Goutsias, J; Mahler, Ronald PS; and Nguyen, HT, eds.: (1998) Random Sets: Theory and Applications, Springer-Verlag

[4] Guan, J and Bell, DA: (1992) Evidence Theory and its Applications, v. 1-2, North Holland

[5] JC Helton, JD Johnson, WL Oberkampf, and CJ Sallaberry: (2004) "Sensitivity Analysis in Conjunction with Evidence Theory Representations of Epistemic Uncertainty", Reliability Engineering and Systems Safety, in press

[6] Helton, JC; Johnson, JD; and Oberkampf, WL: (2004) "An Exploration of Alternative Approaches to the Representation of Uncertainty in Model Predictions", Reliability Engineering and Systems Safety, v. 95:1-3, pp. 39-72

[7] Helton, JC and Oberkampf, WL: (2004) "Special Issue: Alternative Representations of Epistemic Uncertainty", in: Reliability Engineering and Systems Safety, v. 95:1-3

[8] Joslyn, Cliff and Ferson, Scott: (2004) "Approximate Representations of Random Intervals for Hybrid Uncertainty Quantification", in: 4th Int. Conf. on Sensitivity Analysis of Model Output (SAMO 04), ftp://ftp.c3.lanl.gov/pub/users/joslyn/samo04.pdf

[9] Joslyn, Cliff and Helton, Jon C: (2002) "Bounds on Plausibility and Belief of Functionally Propagated Random Sets", in: Proc. Conf. North American Fuzzy Information Processing Society (NAFIPS 2002), pp. $412-417$

[10] Li, S; Ogura, Y; and Kreinovich, V, eds.: (2003) Limit Theorems and Applications of Set Valued and Fuzzy Valued Random Variables, Kluwer

[11] Oberkampf, WL; Helton, JC; Joslyn, CA; Wojtkiewicz SF, Ferson, S: (2004) "Uncertainty in System Response Given Uncertain Parameters", Reliability Reliability Engineering and System Safety, v. 95:13, pp. $11-20$

[12] Yager, Ronald R: (1986) "Arithmetic and Other Operations on Dempster-Shafer Structures", Int. J. Man-Machine Studies, v. 25, pp. 357-366

[13] Yager, Ronald R; Kacprzyk, J; and Pedrizzi, M, eds.: (1994) Advances in Dempster-Shafer Theory of Evidence, Wiley 


\section{Figures}

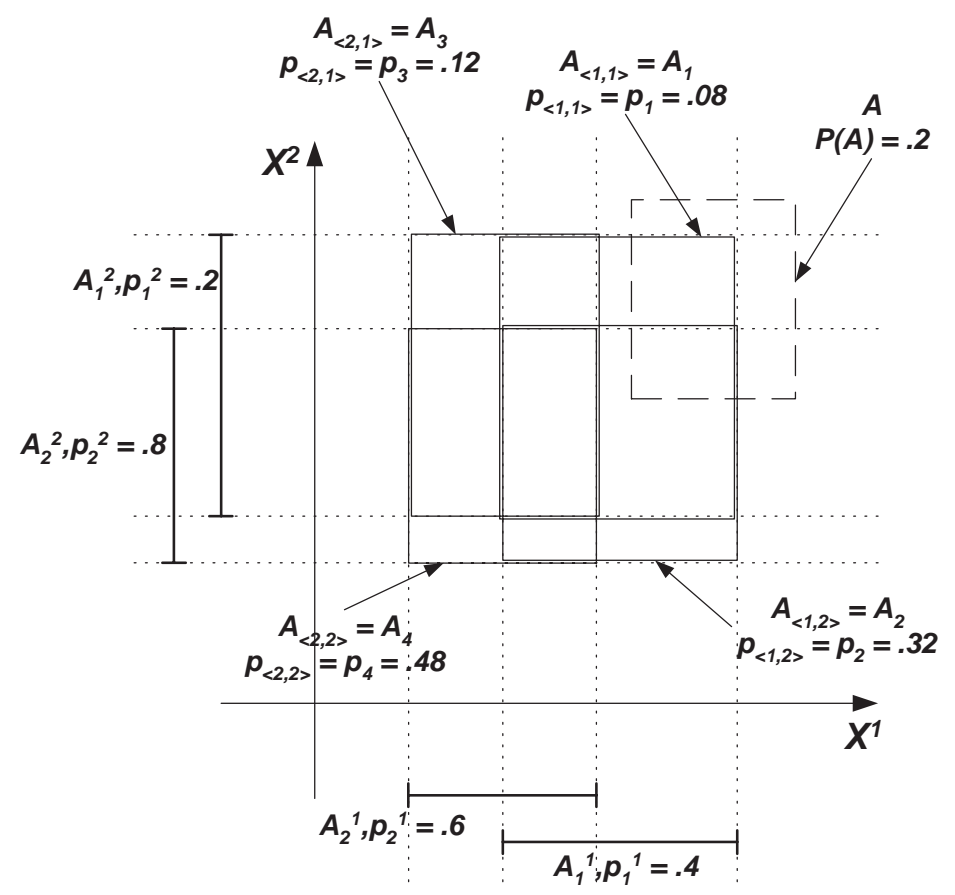

Figure 1: 


\section{Captions}

Figure 1 Example input uncertainty structure $\mathcal{S}$. 